\title{
Optimal Choice of Dividing Surface for the Computation of Quantum Reaction Rates ${ }^{\dagger}$
}

\author{
Cristian Predescu* and William H. Miller \\ Department of Chemistry and Kenneth S. Pitzer Center for Theoretical Chemistry, University of California, \\ Berkeley, California 94720
}

Received: August 30, 2004

\begin{abstract}
We consider the calculation of quantum mechanical rate constants for chemical reactions via algorithms that utilize short-time values of the symmetrized flux - flux correlation function. We argue that the dividing surface that makes optimal use of the short-time quantum information is the surface that minimizes the value at the origin of the symmetrized flux - flux correlation function. We also demonstrate that, in the classical limit, this quantum variational criterion produces the same dividing surface as Wigner's variational principle. Finally, we argue that the quantum variational criterion behaves in a nearly optimal fashion with respect to the minimization of the extent of re-crossing flux.
\end{abstract}

\section{Introduction}

It is well-recognized that the rate constant for a chemical reaction can be written as the time integral of a flux autocorrelation function, ${ }^{1-4}$

$$
k(T) Q_{\mathrm{r}}(T)=\int_{0}^{\infty} C_{\mathrm{F}}(t) \mathrm{d} t
$$

where we write the correlation function first in a slightly more general form than usual,

$$
C_{\mathrm{F}}(t)=\operatorname{tr}\left[\mathrm{e}^{-(\lambda-i t / \hbar) H} \hat{F} \mathrm{e}^{-(\beta-\lambda+i t / \hbar) H} \hat{F}\right]
$$

with the value of $\lambda$ being restricted to the interval $[0, \beta] . Q_{\mathrm{r}}(T)$ is the reactant partition function, per unit volume for a bimolecular reaction. We assume that the Hamiltonian of the system is Cartesian and, for simplicity, that the coordinates are scaled so that the mass is the same for each degree of freedom [i.e., $\left.\hat{H}=\hat{\mathbf{p}}^{2} / 2 m_{0}+V(\hat{\mathbf{x}})\right]$. The flux operator $\hat{F}$ is then given by

$$
\hat{F}=\frac{1}{2 m_{0}}\left[\delta(s(\hat{\mathbf{x}})) \frac{\partial s}{\partial \mathbf{x}} \cdot \hat{\mathbf{p}}+\hat{\mathbf{p}} \cdot \delta(s(\hat{\mathbf{x}})) \frac{\partial s}{\partial \mathbf{x}}\right]
$$

where $\hat{\mathbf{p}}=-i \hbar \partial / \partial \mathbf{x}$ is the usual Cartesian momentum operator and $s(\mathbf{x})$ is a function of coordinates that is negative (positive) on the reactant (product) side. This function defines the dividing surface (DS) via the equation

$$
s(\mathbf{x})=0
$$

By its very construction, the flux operator is Hermitian, i.e., $\hat{F}^{\dagger}$ $=\hat{F}$.

It was pointed out by Miller, Schwartz, and Tromp (MST) ${ }^{3}$ that the rate constant given by eq 1 is independent of the choice of $\lambda$ in the correlation function of eq 2 , though the correlation function itself is not. The early work of Yamamoto ${ }^{1}$ used the Kubo form of the correlation function, which corresponds to averaging eq 2 over $\lambda$ from 0 to $\beta$. MST used a correlation function that corresponds to the choice $\lambda=\beta / 2$. The Kubo form

* To whom correspondence should be addressed. E-mail: cpredescu@ comcast.net.

† Part of the special issue "David Chandler Festschrift". of Yamamoto is sometimes more useful, 5,6 and other times that of MST (but see also the appendix) is more useful. The "nice" feature of the latter is that the Boltzmann operators are combined with the time evolution operators in a symmetric fashion

$$
C_{\mathrm{F}}(t)=\operatorname{tr}\left[\mathrm{e}^{-(\beta / 2-i t / \hbar) H} \hat{F} \mathrm{e}^{-(\beta / 2+i t / \hbar) H} \hat{F}\right]
$$

which has facilitated approximations based on analytic continuation $^{7-10}$ or inverse moment ${ }^{11}$ approaches. For purposes in this paper, it is of central importance that the MST correlation function is finite at $t=0$, whereas the Kubo one diverges there. $^{3,4}$ In the limit $\hbar \rightarrow 0$, the value of the MST correlation function at $t=0$ diverges (as it should) at a rate that is proportional to the classical transition state theory (TST) reaction rate, $k_{\mathrm{cl}, \mathrm{TST}}(T) Q_{\mathrm{r}}(T)$, as described by the equation

$$
C_{\mathrm{F}}(0)=\frac{2}{\hbar \beta} k_{\mathrm{cl}, \mathrm{TST}}(T) Q_{\mathrm{r}}(T)[1+O(\hbar)]
$$

This connection with classical TST is proved in section IV and constitutes one of the arguments demonstrating the suitability of the MST correlation function for the computation of quantum reaction rates.

Another general feature of eqs 1 and 2 is that the rate constant is independent of the location of the DS, although the correlation function itself is not. This is true, of course, only if the quantum dynamics (i.e., the time evolution operators) is generated to times long enough for the integral of $C_{\mathrm{F}}(t)$ to converge. Because of this, some DSs are better than others, for practical purposes. Curve a in Figure 1, for example, shows the sketch of the fluxflux correlation function (of the MST type) for a "direct" reaction, where the DS has been chosen in a location so that there is no "re-crossing flux," that is, so that it is positive for all $t \geq 0$. Curve $\mathrm{b}$ in Figure 1, on the other hand, shows the correlation function with the DS located in a region for which there is re-crossing flux (corresponding classically to trajectories that begin on the DS at $t=0$ and re-cross it at some later time). As noted, the integrals of the two correlation functions in Figure 1 are the same, but the practical advantages of choosing the $\mathrm{DS}$ as in case a are clear, particularly if $C_{\mathrm{F}}(t)$ is calculated by some procedure that has a significant numerical error associated with it (as will almost always be the case). In case b of Figure 


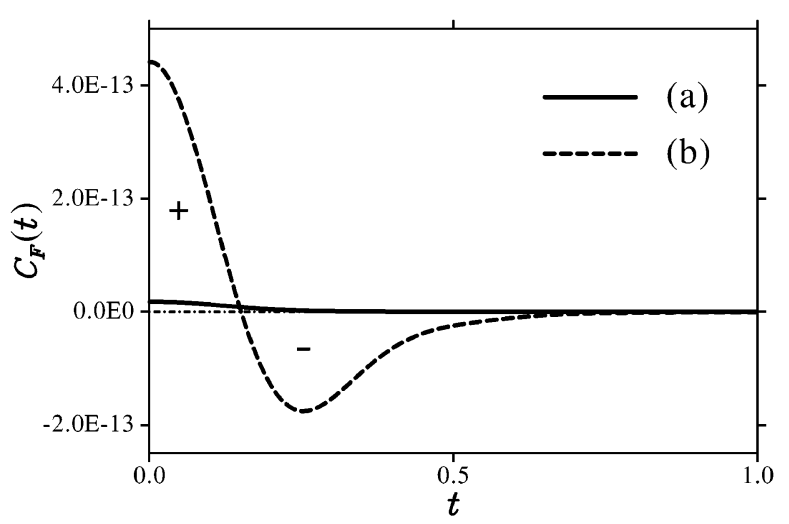

Figure 1. Flux-flux correlation function for a low-temperature onedimensional symmetric Eckart barrier in atomic units, with the time given in units of $\hbar \beta$. The DS for the correlation function in case a (solid line) is located at the top of the barrier. The DS for the correlation function in case $\mathrm{b}$ (dashed line) is displaced from the top by a distance that corresponds to about half of the average barrier thickness.

1, the integral of $C_{\mathrm{F}}(t)$, that is, the rate constant, is given by the difference of two usually very large numbers, the integral of the positive part minus that of the negative part, whereas in case a, there is no such cancellation. In this respect, notice that the magnitude of the correlation function in case b is at least 20 times larger than the magnitude of the correlation function in case a, although the DS is only slightly displaced from the top of the barrier (about half the thickness of the barrier).

The benefit of choosing the DS to eliminate (or at least minimize) the re-crossing flux is even more critical if one uses an approximate rate theory that is based on a short-time approximation to $C_{\mathrm{F}}(t)$ that assumes there is no re-crossing flux, that is, some kind of transition-state-like theory, such as the recent "quantum instanton" approximation ${ }^{12}$ and its extensions. ${ }^{13}$ It is clear that a TST-type approximation would be totally inappropriate for case b of Figure 1, while quite appropriate for the situation in case a. In classical mechanics, these considerations lead to the well-known Wigner variational criterion for choosing the "best" DS, namely, the one which makes the TST rate constant a minimum. ${ }^{14-17}$

The object of this paper is to obtain a criterion for choosing the "best" DS for the quantum mechanical case, under the assumption that only short-time information will be available for $C_{\mathrm{F}}(t)$. Such a criterion corresponds most closely to Wigner's classical variational principle and, in fact, we shall see that Wigner's principle is recovered in the classical limit. To apply to most approaches that avoid the evaluation of the long-time dynamics, the quantum variational criterion is developed starting solely from the assumption that the exact evaluation of the rate of reaction is desired, but only short-time information is available. The rationale behind the quantum criterion is to maximize the contribution of the short-time correlation function to the value of the quantum reaction rate. However, because of this, we expect the variational criterion to produce surfaces of minimal re-crossing flux, for which the long-time dynamics results in nearly negligible values of the flux autocorrelation function at long times. Therefore, such a variational criterion is expected to be useful whether one is actually computing $C_{\mathrm{F}}(t)$ for some finite time or one is using an approximate rate theory based on a short-time approximation that neglects the possibility of re-crossing effects.

To preview our basic result somewhat, we refer again to the sketch in Figure 1. The correlation function with the larger value of $C_{\mathrm{F}}(0)$ has a larger area under its positive region, and so, it must have a larger area under its negative region because the time integrals of the two correlation functions are the same. It is then intuitively clear that the DS with no (or, at least, minimal) re-crossing flux will be the one for which $C_{\mathrm{F}}(0)$ is a minimum, and this is precisely the criterion we propose. The reader may have anticipated our proposal from eq 6 and from the aforementioned requirement that the variational criterion must transform into Wigner's variational principle in the limit $\hbar \rightarrow$ 0 . From the very beginning, we observe that the proposed quantum criterion is quite practical to implement because it involves no real-time dynamics. The goal of the remainder of the paper is to develop this idea as generally and as rigorously as possible.

\section{Motivating the Need for an "Optimal” DS}

As mentioned in the introduction, the reaction rate is invariant to the particular choice of DS, so long as the surface separates reactants from products. The calculation of reaction rates can be greatly simplified, however, if an "optimal" choice for the DS is made. Strictly speaking, the optimal DS is the one that minimizes the computational cost for a particular algorithm that is utilized to evaluate the integral

$$
k(T) Q_{\mathrm{r}}(T)=\int_{0}^{\infty} C_{\mathrm{F}}(t) \mathrm{d} t
$$

Such an optimal DS is useful in practice, of course, only if the computational time necessary to find the surface is not greater than the time necessary to evaluate the right-hand side of eq 7 . It appears that there is no universal definition for the DS, because this surface is algorithm-dependent.

Even so, as we shall see, certain features common to all known algorithms lead to very general criteria for identifying nearly optimal DSs. Because the difficult part in evaluating the integral appearing in eq 7 is the calculation of the correlation function $C_{\mathrm{F}}(t)$ at certain quadrature points $0 \leq t_{1}<t_{2}<\ldots<$ $t_{n}<\infty$, the optimal DS depends on the algorithms utilized to compute the flux autocorrelation function. However, all known algorithms that attempt to evaluate the flux autocorrelation function for arbitrarily long times suffer from the so-called "dynamical sign problem." Thus, the computational time necessary to evaluate $C_{\mathrm{F}}(t)$ with a prescribed absolute error increases at least exponentially with the inverse error as well as with certain physical parameters, such as the time $t \geq 0$ and the dimensionality of the system. ${ }^{18}$

Actually, the flux autocorrelation function can be evaluated in a stable way by means of Monte Carlo imaginary-time pathintegral simulations, but only for times shorter than $\beta \hbar / 2 .{ }^{11}$ As can be demonstrated by analytical continuation ${ }^{19}$ or probabilistic arguments, ${ }^{11}$ the values for longer times and, therefore, the reaction rate are uniquely determined by the values of the fluxflux correlation function on the interval $[0, \beta \hbar / 2]$. However, the errors for the long-time quantities increase exponentially with the errors in the values of the short-time correlation function for all known reconstruction techniques (such reconstruction techniques involve unstable inverse problems, such as inverse real Laplace transforms ${ }^{9,10}$ or inverse moment problems ${ }^{11}$ ).

Given this state of affairs, it becomes apparent that algorithms and DSs that make best use of the available short-time information have certain numerical advantages. At this stage, the sentence "that makes best use of the short-time information" is quite ambiguous, but we shall make definite sense of it in the following section. However, the sentence underlies one of the main assumptions of the present paper: that algorithms relying on the calculation of long-time $(t \geq \hbar \beta / 2)$ quantum 


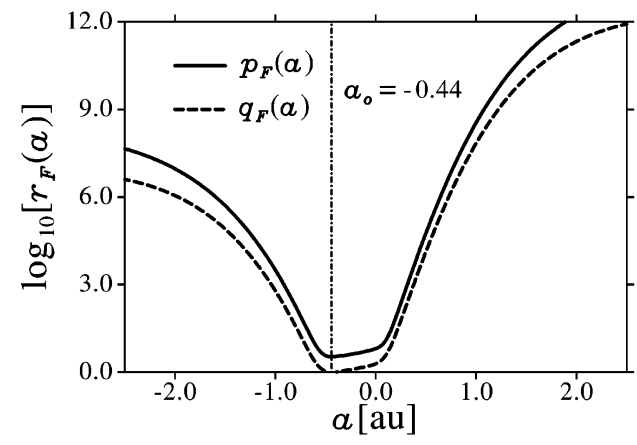

Figure 2. Common (base 10) logarithms of the ratios $p_{\mathrm{F}}(a)$ and $q_{\mathrm{F}}(a)$ [denoted generically by $r_{\mathrm{F}}(a)$ ] as functions of the position $a$ of the DS relative to the top of the barrier, for an asymmetric Eckart barrier at low temperature. Although the two variational criteria discussed in the text are different, they produce virtually identical locations for the optimal DS, in this case $a_{0} \approx-0.44$.

dynamics are less efficient in comparison with those only requiring short-time $(t<\hbar \beta / 2)$ information.

Parenthetically, we observe that, if long-time dynamics were an option, then the obvious choice for a nearly optimal solution would be the surface that minimizes the quantity

$$
\int_{0}^{\infty}\left|C_{\mathrm{F}}(t)\right| \mathrm{d} t=\int_{0}^{\infty} C_{\mathrm{F}}{ }^{+}(t) \mathrm{d} t+\int_{0}^{\infty} C_{\mathrm{F}}{ }^{-}(t) \mathrm{d} t
$$

In eq $8, C_{\mathrm{F}}{ }^{+}(t)$ and $C_{\mathrm{F}}{ }^{-}(t)$ refer to the positive and the negative parts of $C_{\mathrm{F}}(t)$, so that

$$
\int_{0}^{\infty} C_{\mathrm{F}}(t) \mathrm{d} t=\int_{0}^{\infty} C_{\mathrm{F}}{ }^{+}(t) \mathrm{d} t-\int_{0}^{\infty} C_{\mathrm{F}}{ }^{-}(t) \mathrm{d} t
$$

Because the difference given by eq 9 is independent of the DS and because the two terms of the difference are generally evaluated with a fixed relative error only, one must minimize the sum of the two positive terms, to minimize the absolute error of the difference. In this way, the relative error of the difference is also minimized.

The general rule of numerical analysis utilized above is that we must avoid the calculation of various quantities as differences of two positive large numbers, to prevent loss of precision. In this respect, it is worth noting that the ratio

$$
p_{\mathrm{F}}(a)=\int_{0}^{\infty}\left|C_{\mathrm{F}}(t)\right| \mathrm{d} t / \int_{0}^{\infty} C_{\mathrm{F}}(t) \mathrm{d} t
$$

may increase rapidly to very large values upon the variation of the parameters controlling the position and the shape of the DS (parameters denoted collectively by $a$ ), especially at low temperature. The situation is illustrated in Figure 2, where we have plotted the logarithm of the ratio $p_{\mathrm{F}}(a)$ as a function of the displacement $a$, for a low-temperature one-dimensional asymmetric Eckart barrier.

From the discussion in the preceding paragraphs, it is clear that finding the optimal DS is a crucial step in the calculation of any rate of reaction. We have also argued that the minimization of the quantity given by eq 8 leads to a nearly optimal DS. Even so, there is one important objection that must be raised against the use of eq 8: one is required to perform a long-time dynamical simulation anyway, to compute the very functional that is minimized. Although the necessary precision is relative to the value of the integral

$$
\int_{0}^{\infty}\left|C_{\mathrm{F}}(t)\right| \mathrm{d} t
$$

and not to the value of the final reaction rate, the calculation is still not feasible because of the notorious sign problem.

But even for classical systems, there is a zero-time criterion that is a better computational choice than the time integral of the absolute value of the flux autocorrelation function. This criterion is provided by Wigner's variational principle, ${ }^{14-17}$ the computation usefulness of which has long been established by numerous applications. ${ }^{20}$ Wigner's variational principle asserts that the instantaneous flux of particles through any surface separating products from reactants is an upper bound of the true reaction rate. The principle is applied by calculating the TST reaction rate with the DS at different locations. The lowest rate calculated is the best estimate of the true reaction rate, although the latter quantity is not always attained. In the present paper, we suggest that the surface that minimizes the TST rate of reaction is also a nearly optimal DS for the problem of minimizing the cost of computing the integral appearing in eq 7. That Wigner's variational principle leads to computationally nearly optimal DSs can be inferred from the fact that, for those surfaces for which no or minimal re-crossings occur, the most important contribution to the flux-flux correlation function comes from a delta function positioned at $t=0$. In these conditions,

$$
k_{\mathrm{cl}, \mathrm{TST}}(T) Q_{\mathrm{r}}(T) \approx \int_{0}^{\infty}\left|C_{\mathrm{F}}^{\mathrm{cl}}(t)\right| \mathrm{d} t
$$

to a good degree. Therefore, Wigner's variational principle and the variational criterion based on the minimization of the time integral of the absolute value of the flux-flux correlation function should lead to nearly identical optimal DSs. In fact, these optimal DSs are strictly the same for those systems for which the TST theory becomes an exact theory.

But how about quantum systems? As mentioned in the introduction, we propose the minimization of the MST correlation function at $t=0, C_{\mathrm{F}}(0)$, as a criterion likely to produce a nearly optimal DS. To support our proposal through a numerical experiment, let us define the ratio

$$
q_{\mathrm{F}}(a)=C_{\mathrm{F}}(0) / \min _{F} C_{\mathrm{F}}(0)
$$

in analogy to eq 10. As it can be seen from Figure 2, the common logarithm of $q_{\mathrm{F}}(a)$ mimics very closely the behavior of the same quantity for $p_{\mathrm{F}}(a)$. In fact, the two minimization criteria produce almost the same location of the dividing point, namely, $a_{0} \approx-0.44$. At least in the limit $\hbar \rightarrow 0$, the excellent behavior of the optimality criterion based on $C_{\mathrm{F}}(0)$ can be understood via Wigner's variational principle, into which it transforms (as demonstrated in section IV). The argument for the general quantum case is given in the next section.

\section{Rationale for the $C_{\mathrm{F}}(0)$ Quantum Variational Criterion}

In this section, we present the rationale for the quantum variational criterion based on the minimization of the value of the flux autocorrelation function at the origin. We commence with a short summary of the main line of thought. We point out that short-time information is extremely redundant, although it completely determines the values of the flux autocorrelation function at all later times. A more suitable set of short-time data is the sequence of even derivatives at the origin of the correlation function. These derivatives are positive and represent the moments of the spectral function associated with the flux autocorrelation function. We shall assume that we are able to compute these moments with any desired relative accuracy, 
although this assumption is still not sufficient for a reliable determination of the rate of reaction. The reason for the insufficiency is that the rate of reaction is obtained as the result of an inverse moment problem. The solution of such a problem is known to be unstable with respect to small relative errors in the input data. As such, any algorithm designed to approximate the solution of the infinite moment problem by matching a finite number of moments becomes less and less stable in the limit of a large number of matched moments. To compensate for this instability, one is required to increase the accuracy in the determination of the moments, a requirement that underlies the redundancy of the moment information.

For the problem of computing reaction rates, where only the value of the flux-flux spectral function near the origin is important, the redundancy in the moment information and, therefore, the instability of the inverse moment problem are caused by the fact that the high-order moment information comes from frequency regions that are far away from the origin. This state of affairs is independent of the nature of the algorithm utilized for the resolution of the finite moment problem. It follows that the definite way of stabilizing the results for all inversion algorithms is by working with the DS for which the main information contained in the moments comes from frequency regions that are as close as possible to the origin. In this section, we argue that the surface that minimizes the value of the flux -flux correlation function at the origin is optimal in this respect.

It can be shown by analytical continuation ${ }^{19}$ or by probabilistic $\operatorname{arguments}^{11}$ that any short-time information of the autocorrelation function [that is, knowledge of the flux autocorrelation function $C_{\mathrm{F}}(t)$ for all times $0 \leq t \leq \epsilon$ up to an arbitrary $\epsilon>0$ ] uniquely determines the autocorrelation function. Therefore, at least in principle, the absolute rate of reaction can be recovered from such short-time information. Because in the limit $\epsilon \rightarrow 0$ all values of $C_{\mathrm{F}}(t)$ tend to $C_{\mathrm{F}}(0)$ by the continuity of the autocorrelation function at the origin, it is easy to see that the short-time information is highly redundant, that it must be computed with very high accuracy, and that all useful knowledge is actually contained in the way the values of $C_{\mathrm{F}}(t)$ for various times $t \in[0, \epsilon]$ relate to one another. After straightforward algebraic transformations via certain finitedifference schemes, the short-time information can be more suitably used in the form of the sequence of derivatives at the origin of the flux autocorrelation function. In this respect, we mention that it can be proved under quite general assumptions that the flux autocorrelation function is analytical at the origin, with a radius of convergence of $\beta \hbar / 2 .{ }^{11}$ Therefore, without loss of generality, by "short-time information", we shall understand the sequence of derivatives at the origin of the flux autocorrelation function or, equivalently (see eq 15), the sequence of moments of the flux - flux spectral density. This definition has the additional advantage that it no longer requires the utilization of the arbitrary $\epsilon>0$ parameter introduced above.

The spectral density of the flux autocorrelation function is given by the Fourier transform

$$
\bar{C}_{\mathrm{F}}(\omega)=\frac{1}{2 \pi} \int_{\mathbb{R}} \mathrm{e}^{-i \omega t} C_{\mathrm{F}}(t) \mathrm{d} t
$$

which is generally defined as a non-negative distribution, as shown by the identity ${ }^{11}$

$$
\bar{C}_{\mathrm{F}}(\omega)=\hbar \mathrm{e}^{-\beta \omega \hbar / 2} \int_{\mathrm{R}} \mathrm{e}^{-\beta E}|\langle E+\omega \hbar|F| E\rangle|^{2} \mathrm{~d} E
$$

The spectral density is symmetric in the variable $\omega$. Therefore, its odd moments are 0 . Its even moments $D_{2 \mathrm{k}}$ are related to the even-order derivatives of the flux autocorrelation function at the origin, according to the equation

$$
D_{2 \mathrm{k}} \equiv \int_{\mathbb{R}} \bar{C}_{\mathrm{F}}(\omega) \omega^{2 \mathrm{k}} \mathrm{d} \omega=(-1)^{k} \frac{\mathrm{d}^{2 k} C_{\mathrm{F}}}{\mathrm{d} t^{2 k}}(0)
$$

The absolute rate of reaction is related to the value of the spectral density at the origin, as follows:

$$
k(T) Q_{\mathrm{r}}(T)=\int_{0}^{\infty} C_{\mathrm{F}}(t) \mathrm{d} t=\frac{1}{2} \int_{-\infty}^{\infty} C_{\mathrm{F}}(t) \mathrm{d} t=\pi \bar{C}_{\mathrm{F}}(0)
$$

The problem that we are given to solve is a very difficult inverse moment problem: recover the value of the spectral density at the origin $\bar{C}_{\mathrm{F}}(0)$ (the spectral function is assumed continuous near the origin) given the moments of the spectral density. It is no news that such a problem is ill-posed both because the finite moment problem is not uniquely determined and also because the infinite moment problem is not stable with respect to small relative errors in the moment data. The finite moment problem can be regularized by choosing that solution that is physically more relevant or for which the stability of the resulting inverse problem is the largest. However, in the limit that the number of matched moments goes to infinity, all finite inverse problems become unstable, no matter how good the regularization algorithm is. This is so because the infinite moment problem is not stable. The cause of this instability is the redundancy of the moment information.

Redundancy of data is a measure of the lack of sensitivity of the information that is computed with respect to changes in the values of the quantity that is reconstructed. If the information is highly sensitive, then we can easily differentiate between the various values of the quantity to be reconstructed. This means that one may design highly effective inversion algorithms. In mathematical language, the inverse problem has good continuity properties with the input information. On the contrary, if the information is not sensitive to the values of the quantity we want to reconstruct, any inversion algorithm is unstable. In the latter situation, the computed data do not give us radically new information about the object we want to reconstruct. We say that the information provided is redundant. We mention that high redundancy of data sets an intrinsic limit on the resolution capabilities of the inversion algorithms, a limit that cannot be overcome by any numerical tricks.

The redundancy of the moment information with respect to the value of the flux-flux spectral density at the origin can be understood by looking at the values of the normalized positive functions

$$
\bar{C}_{\mathrm{F}}(\omega) \omega^{2 k} / D_{2 k}
$$

for increasing $k$ 's. As illustrated in Figure 3 for the case of a quantum free particle, the main contribution of the values of $\bar{C}_{\mathrm{F}}(\omega)$ to the high-order moments comes from regions that are further and further away from the origin, as the order of the moments increases. This behavior can be explained by observing that the polynomial $\omega^{2 k}$ increases ever more rapidly with $\omega$, as the value of $k$ increases. As illustrated in Figure 4, by varying the location of the DS, the frequency regions that contribute the most to the moment values can be made closer or further from the origin. By making these regions as close as possible to the origin, we increase the sensitivity of the moment information with respect to the value of the flux-flux spectral density at the origin. To move the important regions closer to 


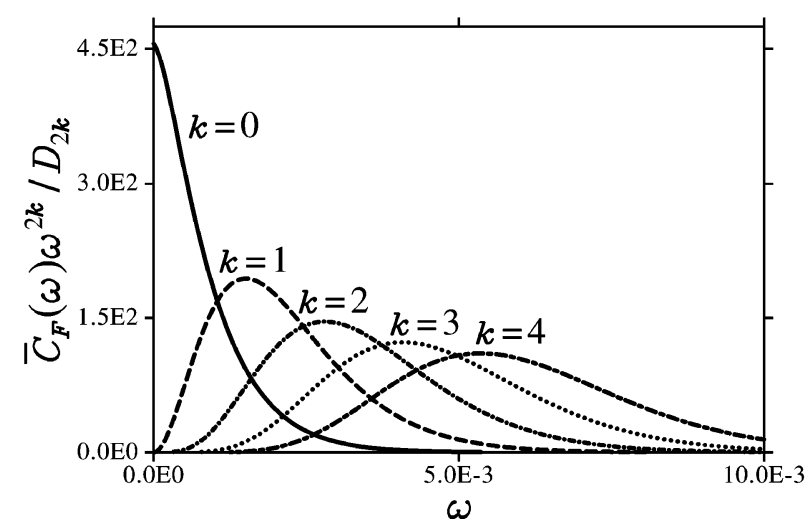

Figure 3. Graph illustrating that the higher-order moments mostly recover information from frequency regions that are further and further away from the origin, as the order $2 k$ of the moments increases. The plot is in atomic units and for a quantum free particle.

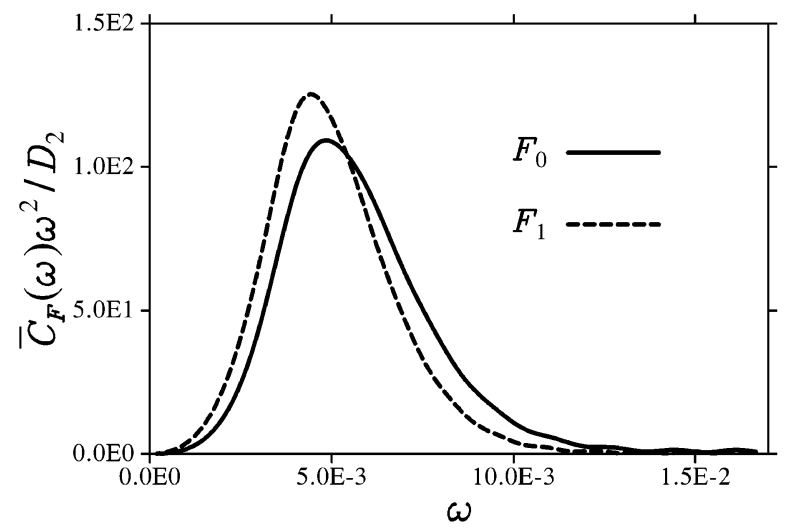

Figure 4. Frequency distributions $\bar{C}_{\mathrm{F}}(\omega) \omega^{2} / D_{2}$ for two DSs for a lowtemperature one-dimensional asymmetric Eckart barrier (the results are in atomic units). The DS $F_{0}$ (solid line) corresponds to the point at the top of the barrier, whereas the DS $F_{1}$ (dashed line) is the one that minimizes the value of the flux autocorrelation function at the origin. The distribution associated with the latter surface is seen to be closer to the origin. Therefore, the contribution of the second moment to the value of the spectral density $\bar{C}_{\mathrm{F}}(\omega)$ near the origin is larger for the surface $F_{1}$.

the origin for moments of all orders, we must increase the importance of $\bar{C}_{\mathrm{F}}(\omega)$ near the origin, as measured relative to the importance of $\bar{C}_{\mathrm{F}}(\omega)$ away from the origin.

In other words, by varying the DS associated with the flux operator $\hat{F}$, we must maximize the value of the ratio of integrals

$$
R_{\epsilon}(\hat{F})=\int_{0}^{\epsilon} \bar{C}_{\mathrm{F}}(\omega) \mathrm{d} \omega / \int_{\epsilon}^{\infty} \bar{C}_{\mathrm{F}}(\omega) \mathrm{d} \omega
$$

for some very small, but as yet undefined, $\epsilon>0$. The more clear mathematical statement is that we must work in the limit $\epsilon \rightarrow 0$. In this respect, we notice that, because the result of the maximization with respect to $\hat{F}$ is not affected by a multiplication with $\epsilon^{-1}$, we may equivalently maximize $\epsilon^{-1} R_{\epsilon}(\hat{F})$, and we shall show that $\lim _{\epsilon \rightarrow 0} \epsilon^{-1} R_{\epsilon}(\hat{F})$ is, indeed, well-defined. To serve our purpose, the limit must exist and have a unique global maximum with respect to the variable $\hat{F}$. If there is more than one global maximum, the degeneracy can be resolved by analyzing the correction term of order $\epsilon$. If the degeneracy is still not completely resolved, one must look to the correction term of order $\epsilon^{2}$, and so on. In this paper, we shall assume that such a degeneracy does not appear. If it does appear in practical applications, we choose one of the maxima randomly.

At this point, it is useful to remember that the spectral density $\bar{C}_{\mathrm{F}}(\omega)$ is continuous at the origin and that its value at the origin

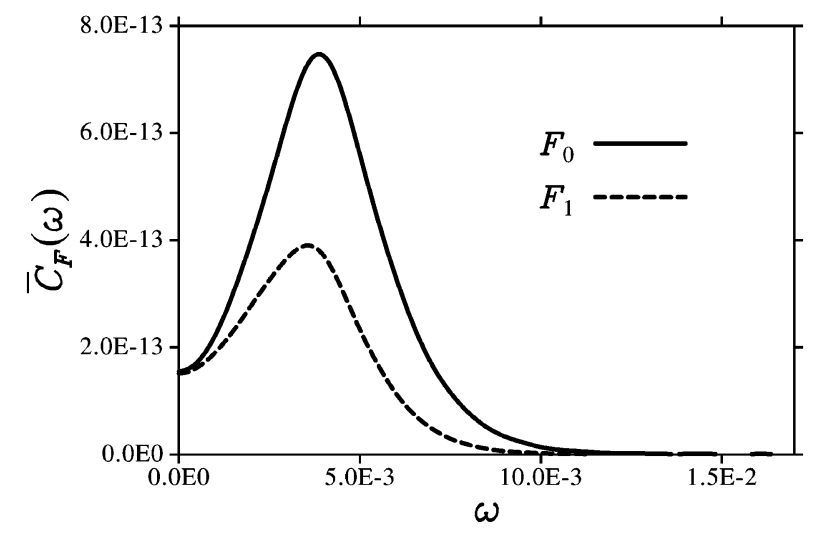

Figure 5. Spectral densities for two DSs for a low-temperature onedimensional asymmetric Eckart barrier (the results are in atomic units). The DS F $\mathrm{F}_{0}$ (solid line) corresponds to the point at the top of the barrier, whereas the DS $F_{1}$ (dashed line) is the one that minimizes the value of the flux autocorrelation function at the origin. In agreement with the fact that the rate of reaction is independent of the location of the DS, the values of the two spectral densities at the origin are the same and equal to $\pi^{-1} k(T) Q_{\mathrm{r}}(T)$.

is independent of the DS. This state of affairs is depicted in Figure 5, where we have plotted the spectral densities for two different DSs for a low-temperature asymmetric Eckart barrier. As a consequence of these properties and of eq 16, we have

$$
\lim _{\epsilon \rightarrow 0} \frac{1}{\epsilon} \int_{0}^{\epsilon} \bar{C}_{\mathrm{F}}(\omega) \mathrm{d} \omega=\bar{C}_{\mathrm{F}}(0)=k(T) Q_{\mathrm{r}}(T) / \pi
$$

for all DSs. Next, we compute

$$
\begin{aligned}
\lim _{\epsilon \rightarrow 0} \epsilon^{-1} R_{\epsilon}(\hat{F}) & =\frac{k(T) Q_{\mathrm{r}}(T)}{\pi} / \int_{0}^{\infty} \bar{C}_{\mathrm{F}}(\omega) \mathrm{d} \omega \\
& =\frac{2}{\pi} \frac{k(T) Q_{\mathrm{r}}(T)}{C_{\mathrm{F}}(0)}
\end{aligned}
$$

where, for the last equality, we have utilized the Fourier identity

$$
\int_{0}^{\infty} \bar{C}_{\mathrm{F}}(\omega) \mathrm{d} \omega=\frac{1}{2} C_{\mathrm{F}}(0)
$$

Equation 17 represents the main result of the section. It shows that the surface that minimizes the redundancy of the moment information with respect to the evaluation of the quantum rate of reaction is the surface that minimizes the value of the flux autocorrelation function at the origin. This conclusion is independent of the nature of the algorithms utilized for the regularization and inversion of the finite moment problem.

Figure 5 also explains the observation made in the second part of section II that the quantum variational criterion may prove useful for all quantum algorithms, including those that explicitly evaluate the correlation function for times longer than $\beta \hbar / 2$. The frequencies of the main re-crossing events are given by the nonzero maxima of the flux-flux spectral density. By diminishing the weight of the high-frequency part of the flux -flux spectral density, the amplitude of the re-crossing flux is also diminished. Therefore, we expect that the value of the $p_{\mathrm{F}}(a)$ ratio defined by eq 10 and which is a direct measure of the extent of re-crossings is also diminished, for most surfaces.

Finally, let us notice that, because the value at the origin of the spectral function is independent of the choice of the DS, we could minimize any positive combination of 
moments of the form

$$
\int_{\mathbb{R}} \bar{C}_{\mathrm{F}}(\omega)\left(\sum_{k=0}^{n} a_{k} \omega^{k}\right)^{2} \mathrm{~d} \omega
$$

By a judicious choice of parameters $a_{k}$, we could selectively target any frequency region from the spectral function that is particularly troublesome. The problem with this line of reasoning is that we do not know a priori the location of such frequency regions. To borrow a term from information theory, the choice $\left(\sum_{k=0}^{n} a_{k} \omega^{k}\right)^{2}=1$ is the least committed with respect to the exact location of the troublesome frequency regions and is consistent with the hypothesis that no prior knowledge on the structure of the spectral function is given.

\section{Classical Limit of $C_{\mathrm{F}}(0)$}

In this section, we demonstrate that, in the classical limit, Wigner's variational principle and the quantum variational criterion based on the minimization of $C_{\mathrm{F}}(0)$ produce the same optimal surfaces. We interpret the agreement between the two variational approaches as additional evidence in favor of the quantum variational criterion. After all, the computational usefulness of Wigner's variational principle has been well established in practice in countless numerical applications.

For definiteness, we again assume that the physical coordinates have been rescaled such that all masses are equal to the common value $m_{0}$. The value at the origin of the flux autocorrelation function reads

$$
\begin{aligned}
& C_{\mathrm{F}}(0)= \frac{1}{2}\left(\frac{\hbar}{m_{0}}\right)^{2} \sum_{j, k=1}^{d} \int_{\mathbb{R}^{d}} \mathrm{~d} \mathbf{x} \int_{\mathbb{R}^{d}} \mathrm{~d} \mathbf{y} \delta(s(\mathbf{x})) \delta(s(\mathbf{y})) \times \\
& \frac{\partial s}{\partial x_{j}}(\mathbf{x}) \frac{\partial s}{\partial y_{k}}(\mathbf{y})\left[\rho(\mathbf{x}, \mathbf{y} ; \beta / 2) \frac{\partial^{2} \rho}{\partial x_{j} \partial y_{k}}(\mathbf{x}, \mathbf{y} ; \beta / 2)-\right. \\
&\left.\frac{\partial \rho}{\partial x_{j}}(\mathbf{x}, \mathbf{y} ; \beta / 2) \frac{\partial \rho}{\partial y_{k}}(\mathbf{x}, \mathbf{y} ; \beta / 2)\right]
\end{aligned}
$$

where

$$
\rho(\mathbf{x}, \mathbf{y} ; \beta / 2)=\left\langle\mathbf{x}\left|\mathrm{e}^{-\beta H / 2}\right| \mathbf{y}\right\rangle
$$

is the quantum mechanical density matrix.

Let us consider the $d$-dimensional Feynman-Kac formula ${ }^{21,22}$

$$
\begin{aligned}
& \rho(\mathbf{x}, \mathbf{y} ; \beta / 2)= \\
& \quad \rho_{\mathrm{fp}}(\mathbf{x}, \mathbf{y} ; \beta / 2) \mathbb{E} \exp \left\{-(\beta / 2) \int_{0}^{1} V\left[\mathbf{x}_{\mathrm{r}}(\mathrm{u})+\sigma B_{\mathrm{u}}{ }^{0}\right] d \mathrm{u}\right\}
\end{aligned}
$$

which expresses the density matrix as the expected value of a functional of the standard $d$-dimensional Brownian bridge $B_{\mathrm{u}}{ }^{0}$. In eq 20, $\mathbf{x}_{\mathrm{r}}(u)=\mathbf{x}+(\mathbf{y}-\mathbf{x}) u$ and $\sigma=\left(\hbar^{2} \beta / 2 m_{0}\right)^{1 / 2}$, whereas $\rho_{\mathrm{fp}}(\mathbf{x}, \mathbf{y} ; \beta / 2)$ stands for the density matrix of a similar free particle at the inverse temperature $\beta / 2$. With the help of the Feynman-Kac formula, we compute

$$
\begin{array}{r}
\frac{1}{2}\left(\frac{\hbar}{m_{0}}\right)^{2}\left[\rho(\mathbf{x}, \mathbf{y} ; \beta / 2) \frac{\partial^{2} \rho}{\partial x_{j} \partial y_{k}}(\mathbf{x}, \mathbf{y} ; \beta / 2)-\right. \\
\left.\frac{\partial \rho}{\partial x_{j}}(\mathbf{x}, \mathbf{y} ; \beta / 2) \frac{\partial \rho}{\partial y_{k}}(\mathbf{x}, \mathbf{y} ; \beta / 2)\right]= \\
\rho_{\mathrm{fp}}(\mathbf{x}, \mathbf{y} ; \beta / 2)^{2} \mathbb{E} \mathbb{E}^{\prime} \exp \left(-\frac{\beta}{2}\left\{\int_{0}^{1} V\left[\mathbf{x}_{\mathrm{r}}(u)+\sigma B_{u}{ }^{0}\right] \mathrm{d} u+\right.\right. \\
\left.\left.\int_{0}^{1} V\left[\mathbf{x}_{\mathrm{r}}(u)+\sigma B_{u}{ }^{0 \prime}\right] \mathrm{d} u\right\}\right) \mathscr{F}_{j, k}\left(\mathbf{x}, \mathbf{y}, B_{*}{ }^{0}, B_{*}{ }^{0 \prime}\right)
\end{array}
$$

where

$$
\begin{gathered}
\mathscr{F}_{j, k}\left(\mathbf{x}, \mathbf{y}, B_{*}{ }^{0}, B_{*}{ }^{0 \prime}\right)=\frac{1}{2}\left(\frac{\hbar}{m_{0}}\right)^{2}\left\{\frac { \beta } { 2 } \int _ { 0 } ^ { 1 } \left(\partial_{j} V\left[\mathbf{x}_{r}(u)+\sigma B_{\mathrm{u}}{ }^{0 \prime}\right]-\right.\right. \\
\left(\frac{\left.\partial_{j} V\left[\mathbf{x}_{\mathrm{r}}(u)+\sigma B_{\mathrm{u}}{ }^{0}\right]\right)(1-u) \mathrm{d} u \times}{\sigma^{2}}+\frac{\beta}{2} \int_{0}^{1} \partial_{k} V\left[\mathbf{x}_{\mathrm{r}}(u)+\sigma{B_{\mathrm{u}}}^{0 \prime}\right] u \mathrm{~d} u\right)+ \\
\frac{\left.\delta_{j k}-\frac{\beta}{2} \int_{0}^{1} \partial_{j, k}{ }^{2} V\left[\mathbf{x}_{\mathrm{r}}(u)+\sigma B_{\mathrm{u}}{ }^{0 \prime}\right] u(1-u) \mathrm{d} u\right\}}{}
\end{gathered}
$$

The symbols $\mathbb{E}$ and $\mathbb{E}^{\prime}$ in eq 21 denote expected values with respect to the independent $d$-dimensional Brownian bridges $B_{\mathrm{u}}{ }^{0}$ and $B_{\mathrm{u}}{ }^{\prime \prime}$, respectively. The symbol $\delta_{\mathrm{jk}}$ in eq 22 is the Kronecker delta. Remembering the defining equation $\sigma^{2}=\hbar^{2} \beta /\left(2 m_{0}\right)$, it is a simple exercise to demonstrate that the limit $\hbar \rightarrow 0$ of eq 22 is

$$
\lim _{\hbar \rightarrow 0} \mathscr{F}_{j, k}\left(\mathbf{x}, \mathbf{y}, B_{*}{ }^{0}, B_{*}{ }^{0}\right)=\frac{1}{m_{0} \beta} \delta_{j k}
$$

Thus, in the classical limit, the left-hand side of eq 21 is well approximated by

$$
\frac{\delta_{j k}}{m_{0} \beta} \rho(\mathbf{x}, \mathbf{y} ; \beta / 2)^{2}
$$

It follows that the dominant term of the right-hand side of eq 19 , in the limit $\hbar \rightarrow 0$, is

$$
\begin{aligned}
C_{\mathrm{F}}(0) \approx \frac{1}{m_{0} \beta} \sum_{j=1}^{d} \int_{\mathbb{R}^{d}} \mathrm{~d} \mathbf{x} \int_{\mathbb{R}^{d}} \mathrm{~d} \mathbf{y} \delta(s(\mathbf{x})) \delta(s(\mathbf{y})) \times \\
\frac{\partial s}{\partial x_{j}}(\mathbf{x}) \frac{\partial s}{\partial y_{j}}(\mathbf{y}) \rho(\mathbf{x}, \mathbf{y} ; \beta / 2)^{2}
\end{aligned}
$$

Again, in the classical limit,

$$
\begin{gathered}
\rho(\mathbf{x}, \mathbf{y} ; \beta / 2)^{2} \approx\left[\rho_{\mathrm{fp}}(\mathbf{x}, \mathbf{y} ; \beta / 2) \mathrm{e}^{-\beta[V(\mathbf{x})+V(\mathbf{y})] / 4}\right]^{2} \\
=\left(\frac{m_{0}}{2 \pi \hbar^{2} \beta}\right)^{\mathrm{d} / 2} \mathrm{e}^{-\beta[V(\mathbf{x})+V(\mathbf{y})] / 2} \rho_{\mathrm{fp}}(\mathbf{x}, \mathbf{y} ; \beta / 4)
\end{gathered}
$$

which, upon replacement in eq 24 , produces the following dominant term for the value of the flux autocorrelation function:

$$
\begin{aligned}
& C_{\mathrm{F}}(0) \approx \frac{1}{m_{0} \beta}\left(\frac{m_{0}}{2 \pi \hbar^{2} \beta}\right)^{d / 2} \sum_{j=1}^{d} \int_{\mathbb{R}^{d}} \mathrm{~d} \mathbf{x} \int_{\mathbb{R}^{d}} \mathrm{~d} \mathbf{y} \times \\
& \mathrm{e}^{-\beta[V(\mathbf{x})+V(\mathbf{y})] / 2} \delta(s(\mathbf{x})) \delta(s(\mathbf{y})) \frac{\partial s}{\partial x_{j}}(\mathbf{x}) \frac{\partial s}{\partial y_{j}}(\mathbf{y}) \rho_{\mathrm{fp}}(\mathbf{x}, \mathbf{y} ; \beta / 4)
\end{aligned}
$$

We now perform the substitution $\mathbf{y}=\mathbf{x}+\mathbf{z}$ and notice that

$$
\rho_{\mathrm{fp}}(0, \mathbf{z} ; \beta / 4) \rightarrow \delta(\mathbf{z})
$$

in the limit $\hbar \rightarrow 0$. Thus, the contribution to the integral over $\mathbf{z}$ in the expression

$$
\begin{gathered}
C_{\mathrm{F}}(0) \approx \frac{1}{m_{0} \beta}\left(\frac{m_{0}}{2 \pi \hbar^{2} \beta}\right)^{d / 2} \sum_{j=1}^{d} \int_{\mathbb{R}^{d}} \mathrm{~d} \mathbf{x} \int_{\mathbb{R}^{d}} \mathrm{~d} \mathbf{z} \times \\
\mathrm{e}^{-\beta[V(\mathbf{x})+V(\mathbf{x}+\mathbf{z})] / 2} \delta(s(\mathbf{x})) \delta(s(\mathbf{x}+\mathbf{z})) \\
\frac{\partial s}{\partial x_{j}}(\mathbf{x}) \frac{\partial s}{\partial x_{j}}(\mathbf{x}+\mathbf{z}) \rho_{\mathrm{fp}}(0, \mathbf{z} ; \beta / 4)
\end{gathered}
$$


comes from a small region around the point $\mathbf{z}=0$. In the limit $\hbar \rightarrow 0$, because of the identity expressed by eq 26 , all smooth functions that participate at the average over $\mathbf{z}$ in eq 27 can be replaced with their value for $\mathbf{z}=0$. Also, by observing that the norm $\|\mathbf{z}\|$, which enters the expression for $\rho_{\mathrm{fp}}(0, \mathbf{z} ; \beta / 4)$, is invariant to a rotation, we may assume that the reference frame for the variable $\mathbf{z}$ is such that $z_{1}$ is along $\nabla s(\mathbf{x})$, the gradient of $s$ at $\mathbf{x}$.

The only nonsmooth function involving the variable $\mathbf{z}$ is the delta function $\delta(s(\mathbf{x}+\mathbf{z}))$, which can be replaced by

$$
\delta(s(\mathbf{x}+\mathbf{z})) \approx \delta\left(s(\mathbf{x})+\|\nabla s(\mathbf{x})\| z_{1}\right)
$$

Computing the integrals over $z_{2}, z_{3}, \ldots, z_{\mathrm{d}}$ is now an easy task, and eq 27 becomes

$$
\begin{aligned}
C_{\mathrm{F}}(0) & \approx \frac{1}{m_{0} \beta}\left(\frac{m_{0}}{2 \pi \hbar^{2} \beta}\right)^{d / 2} \int_{\mathbb{R}^{d}} \mathrm{~d} \mathbf{x} \mathrm{e}^{-\beta V(\mathbf{x})} \delta(s(\mathbf{x})) \\
& \|\nabla s(\mathbf{x})\|^{2} \int_{\mathbb{R}^{d}} \mathrm{~d} z_{1} \delta\left(s(\mathbf{x})+\|\nabla s(\mathbf{x})\| z_{1}\right) \mathrm{e}^{-z_{1}^{2} / \sigma^{2}} / \sqrt{\pi \sigma^{2}}
\end{aligned}
$$

The integral over $z_{1}$ can be performed with the help of the substitution $y=\|\nabla s(\mathbf{x})\|_{z_{1}}$, as follows

$$
\begin{gathered}
\int_{\mathbb{R}} \mathrm{d} z_{1} \delta\left(s(\mathbf{x})+\|\nabla s(\mathbf{x})\| z_{1}\right) \mathrm{e}^{-z_{1}^{2} / \sigma^{2}} / \sqrt{\pi \sigma^{2}}= \\
\int_{\mathbb{R}} \mathrm{d} y \frac{\delta(s(\mathbf{x})+y)}{\|\nabla s(\mathbf{x})\| \sqrt{\pi \sigma^{2}}} \exp \left(-\frac{y^{2}}{\|\nabla s(\mathbf{x})\|^{2} \sigma^{2}}\right) \\
=\frac{1}{\|\nabla s(\mathbf{x})\| \sqrt{\pi \sigma^{2}}} \exp \left[-\frac{s(\mathbf{x})^{2}}{\|\nabla s(\mathbf{x})\|^{2} \sigma^{2}}\right]
\end{gathered}
$$

Replacing the last result in eq 28 and remembering that $\sigma^{2}=$ $\hbar^{2} \beta /\left(2 m_{0}\right)$, we obtain

$$
\begin{aligned}
C_{\mathrm{F}}(0) \approx \frac{2}{m_{0} \beta}\left(\frac{m_{0}}{2 \pi \hbar^{2} \beta}\right)^{(d+1) / 2} \int_{\mathbb{R}^{d}} \mathrm{~d} \mathbf{x} \mathrm{e}^{-\beta V(\mathbf{x})} \\
\delta(s(\mathbf{x}))\|\nabla s(\mathbf{x})\| \exp \left[-\frac{s(\mathbf{x})^{2}}{\|\nabla s(\mathbf{x})\|^{2} \sigma^{2}}\right]
\end{aligned}
$$

In eq 29, the variable $\mathbf{x}$ is constrained to stay on the surface of equation $s(\mathbf{x})=0$ by the delta function $\delta(s(\mathbf{x}))$. Therefore, the value of the exponential

$$
\exp \left[-\frac{s(\mathbf{x})^{2}}{\|\nabla s(\mathbf{x})\|^{2} \sigma^{2}}\right]
$$

on this surface is always 1. Utilizing the last observation, we end up with the surface integral

$C_{\mathrm{F}}(0) \approx \frac{2}{m_{0} \beta}\left(\frac{m_{0}}{2 \pi \hbar^{2} \beta}\right)^{(d+1) / 2} \int_{\mathbb{R}^{d}} \mathrm{~d} \mathbf{x} \mathrm{e}^{-\beta V(\mathbf{x})} \delta(s(\mathbf{x}))\|\nabla s(\mathbf{x})\|$

Comparing the last integral with the expression of the TST rate constant

$$
\begin{aligned}
k_{\mathrm{cl}, \mathrm{TST}}(T) Q_{\mathrm{r}}(T)=\frac{k_{\mathrm{B}} T}{h}\left(\frac{m_{0}}{2 \pi \hbar^{2} \beta}\right)^{(d-1) / 2} & \\
& \int_{\mathbb{R}^{d}} \mathrm{~d} \mathbf{x} \mathrm{e}^{-\beta V(\mathbf{x})} \delta(s(\mathbf{x}))\|\nabla s(\mathbf{x})\|
\end{aligned}
$$

and using the equality $k_{\mathrm{B}} T / h=1 /(2 \pi \hbar \beta)$, we obtain that

$$
C_{\mathrm{F}}(0) \approx \frac{2}{\hbar \beta} k_{\mathrm{cl}, \mathrm{TST}}(T) Q_{\mathrm{r}}(T)
$$

in the classical limit. The exact mathematical formulation of eq 32 is

$$
\lim _{\hbar \rightarrow 0} \frac{\hbar \beta}{2} \frac{C_{\mathrm{F}}(0)}{k_{\mathrm{cl}, \mathrm{TST}}(T) Q_{\mathrm{r}}(T)}=1
$$

where the dependence with $\hbar$ of $C_{\mathrm{F}}(0)$ and $Q_{\mathrm{r}}(T)$ is implicitly understood.

Equation 32 proves our claim that both the quantum variational criterion based on the minimization of $C_{\mathrm{F}}(0)$ and Wigner's variational principle lead to the same DS in the classical limit. As observed in section III for a similar situation, if there are degeneracies of the optimal DS at the level of the Wigner's variational principle, then these degeneracies might be lifted at the level of the quantum theory. One just needs to take into account the correction terms of order $\hbar^{0} Q_{\mathrm{r}}(T), \hbar^{1} Q_{\mathrm{r}}(T)$, and so forth in eq 32 until the degeneracies are completely lifted or until the list of correction terms is exhausted. However, such an analysis is of little interest and will not be considered. As is apparent from eq 32, the dominant term in the expression of $C_{\mathrm{F}}(0) / Q_{\mathrm{r}}(T)$ is of order $\hbar^{-1}$ and diverges in the limit $\hbar \rightarrow 0$. This finding is in agreement with the well-known properties of the classical flux - flux correlation functions of behaving like $2 k_{\mathrm{cl}, \mathrm{TST}}(T) Q_{\mathrm{r}}(T) \delta(t)$ near the origin and, thus, being infinite at $t$ $=0$.

\section{Summary and Conclusions}

The reader should understand that we have not attempted to develop a quantum TST. Rather, our purpose has been to find the DS that makes best use of short-time information, with the understanding that suitable approximations or exact rates, but not necessarily upper bounds, are sought. On one hand, we have argued that algorithms based on the evaluation of long-time dynamics are inherently unfeasible. On the other hand, we have pointed out that the short-time information is highly redundant and leads to numerically unstable algorithms. We have then asked the question of what is the DS that makes best use of the available short-time information, in the sense of alleviating the numerical instability.

We have concluded that, independent of the nature of the inversion algorithms, the surface that minimizes the value of the MST flux-flux correlation function at $t=0$ is nearly optimal and ensures low redundancy in the input data. To further support the optimality of our DS, we have demonstrated that the quantum variational criterion introduced in the present paper transforms into Wigner's variational principle in the classical limit. This finding is relevant, because Wigner's variational principle is generally considered as making best use of shorttime information, for classical systems.

We have explained that the variational criterion is expected to be useful for all algorithms, including those that explicitly evaluate the correlation function for times longer than $\beta \hbar / 2$. This has been demonstrated in the second part of section II, for an asymmetric Eckart barrier. The usefulness claimed above can be understood in the classical limit via Wigner's variational principle: surfaces that minimize the TST rate of reaction are, to a large extent, also surfaces of minimal re-crossings [as measured by the ratio $p_{\mathrm{F}}(a)$ introduced in section II]. The quantum argument is as follows: because the quantum variational criterion decreases the weight of the high-frequency part of the flux-flux spectral density (the nonzero maxima of which 
are associated with the main re-crossing frequencies), the amplitude of the re-crossing flux is also diminished.

Among all variational criteria that minimize the redundancy of the input data by diminishing the weight of the high-frequency part of the spectral function [see eq 18], the value of the fluxflux correlation function at time zero is the least committed with respect to the location of the troublesome frequency regions. It is, therefore, the only choice consistent with the hypothesis that no prior knowledge on the structure of the spectral function is given.

The arguments presented in the paper allow us to conclude with reasonable certainty that the DS produced by our criterion [i.e., minimization of $\left.C_{\mathrm{F}}(0)\right]$ is nearly optimal for any quantum TST-like theory. The only stringent requirement of such a theory is that the spectral function of the trial correlation function be positive, property exhibited by the exact correlation function and on which the development of our variational criterion has relied. One can, thus, focus future theoretical effort on the development of such theories.

Acknowledgment. This work was supported in part by the National Science Foundation Grant CHE-0345280, the Director, Office of Science, Office of Basic Energy Sciences, Chemical Sciences, Geosciences, and Biosciences Division, U.S. Department of Energy under Contract No. DE AC03-65SF00098, and the U.S.-Israel Binational Science Foundation Award No. 2002170 .

\section{Appendix A: Best Choice of Correlation Function}

The reader may feel uncomfortable with the idea that the best DS is related to the MST correlation function. After all, the computational principle employed for the derivation of the variational criterion is quite general and can be utilized in conjunction with other possible correlation functions, with different results. Which one is the better computational choice? In this appendix, we show that the computational principle utilized to justify the choice of DS also uniquely determines the MST correlation function as the optimal one among all possible choices.

The most general correlation function that may be used is of the form

$$
C_{\mathrm{F}, v}(t)=\int_{0}^{\beta} \operatorname{tr}\left[\mathrm{e}^{-(\lambda-i t / \hbar) H} \hat{F} \mathrm{e}^{-(\beta-\lambda+i t / \hbar) H} \hat{F}\right] \mathrm{d} v(\lambda)
$$

where $\mathrm{d} v(\lambda)$ is a measure on the interval $[0,1]$ that is positive and integrates to 1 [a probability measure, so that the righthand side of eq A1 denotes an average]. For example, for the Yamamoto correlation function, we have $\mathrm{d} \nu(\lambda)=(1 / \beta) \mathrm{d} \lambda$, whereas for the MST correlation function, we have $\mathrm{d} v(\lambda)=$ $\delta(\lambda-\beta / 2) \mathrm{d} \lambda$, that is, a Dirac delta function about $\beta / 2$.

The positivity of the measure $\mathrm{d} v(\lambda)$ is required to ensure positivity of the associated spectral function

$$
C_{\mathrm{F}, \nu}(\omega)=\hbar \int_{0}^{\beta} \mathrm{d} v(\lambda) \mathrm{e}^{-\lambda \omega \hbar} \int_{\mathbb{R}} \mathrm{e}^{-\beta E}|\langle E+\omega \hbar|F| E\rangle|^{2} d \mathrm{E}
$$

expression that can be deduced by simple manipulations, starting with the Dirac identity

$$
e^{-\beta H}=\int_{\mathbb{R}} \mathrm{e}^{-\beta E}|E\rangle\langle E| \mathrm{d} E
$$

The reaction rate does not depend on the choice of the measure $\mathrm{d} v(\lambda)$, because in all cases we have

$$
\begin{aligned}
k(T) Q_{\mathrm{r}}(T)=\int_{0}^{\infty} C_{\mathrm{F}, v}(t) \mathrm{d} t= & \pi C_{\mathrm{F}, v}(0)= \\
& \pi \hbar \int_{\mathbb{R}} \mathrm{e}^{-\beta E}|\langle E|F| E\rangle|^{2} \mathrm{~d} E
\end{aligned}
$$

Thus, all the spectral functions of the type given by eq A2 have a fixed value at the origin, independent of the choice of DS $F$ and measure $\mathrm{d} v(\lambda)$. Accordingly, the spectral function that is computationally most useful is the one that is most localized near the origin, that is, the one that minimizes the value of

$$
\int_{\mathbb{R}} C_{\mathrm{F}, v}(\omega) \mathrm{d} \omega=C_{\mathrm{F}, v}(0)
$$

The minimization with respect to the measure $\mathrm{d} v(\lambda)$ is straightforward. Define

$$
A(\lambda)=\int_{\mathbb{R}} \mathrm{e}^{-\lambda \omega \hbar} C_{\mathrm{F}}^{\mathrm{MST}}(\omega) \mathrm{d} \omega
$$

and notice that

$$
\begin{array}{r}
\int_{\mathbb{R}} C_{\mathrm{F}, \nu}(\omega) \mathrm{d} \omega=\int_{0}^{\beta} \mathrm{d} v(\lambda) \int_{\mathbb{R}} \mathrm{e}^{-(\lambda-\beta / 2) \omega \hbar} C_{\mathrm{F}}^{\mathrm{MST}}(\omega) \mathrm{d} \omega= \\
\int_{-\beta / 2}^{\beta / 2} \mathrm{~d} v(\lambda+\beta / 2) A(\lambda)
\end{array}
$$

Because the MST correlation function is symmetric, by a change of variable $\omega^{\prime}=-\omega$ performed in eq A5, it follows that the function $A(\lambda)$ is also symmetric, that is,

$$
A(\lambda)=A(-\lambda)
$$

Moreover,

$$
\frac{\mathrm{d}^{2}}{\mathrm{~d} \lambda^{2}} A(\lambda)=\hbar^{2} \int_{\mathbb{R}} \mathrm{e}^{-\lambda \omega \hbar} \omega^{2} C_{\mathrm{F}}^{\mathrm{MST}}(\omega) \mathrm{d} \omega>0
$$

is strictly positive, and, therefore, $A(\lambda)$ is a strictly convex function symmetric about origin. It follows the $A(\lambda)$ has a unique global minimum at the origin. Equation A6 then shows that the optimal probability measure $\mathrm{d} v(\lambda+\beta / 2)$ is

$$
\mathrm{d} v(\lambda+\beta / 2)=\delta(\lambda) \mathrm{d} \lambda
$$

or, equivalently,

$$
\mathrm{d} \nu(\lambda)=\delta(\lambda-\beta / 2) \mathrm{d} \lambda
$$

Thus, we have proven that the MST correlation function has the spectral density that is the most localized at the origin among the correlation functions of the type given by eq A1. In particular, the MST correlation function appears to be more suitable for the purpose of computing the quantum reaction rate than the Yamamoto correlation function. Again, the general rule is that, whenever the value of a set of spectral functions at a particular point is independent upon the choice of certain parameters, the spectral function that is most localized around the particular point must be utilized to optimally recover the common value of the spectral functions at that point.

\section{References and Notes}

(1) Yamamoto, T. J. Chem. Phys. 1960, 33, 281.

(2) Miller, W. H. J. Chem. Phys. 1974, 61, 1823

(3) Miller, W. H.; Schwartz, S. D.; Tromp, J. W. J. Chem. Phys. 1983, $79,4889$.

(4) Miller, W. H. J. Phys. Chem. A 1998, 102, 793.

(5) Voth, G. A.; Chandler, D.; Miller, W. H. J. Phys. Chem. 1989, 93 7009 .

(6) Craig, I. R.; Manalopoulos, D. E. J. Chem. Phys., in press.

(7) Thirumalai, D.; Berne, B. J. J. Chem. Phys. 1983, 79, 5029. 
(8) Yamashita, K.; Miller, W. H. J. Chem. Phys. 1985, 82, 5475.

(9) Rabani, E.; Krilov, G.; Berne, B. J. J. Chem. Phys. 2000, 112, 2605.

(10) Sim, E.; Krilov, G.; Berne, B. J. J. Phys. Chem. A 2001, 105, 2824.

(11) Predescu, C. Phys. Rev. E 2004, 70, 066705.

(12) Miller, W. H.; Zhao, Y.; Ceotto, M.; Yang, S. J. Chem. Phys. 2003, $119,1329$.

(13) Miller, W. H.; Ceotto, M.; Yang, S. J. Chem. Phys., in press.

(14) Wigner, E. P. J. Chem. Phys. 1937, 5, 720

(15) Wigner, E. P. Trans. Faraday Soc. 1938, 34, 29.
(16) Keck, J. C. Adv. Chem. Phys. 1967, 13, 85.

(17) Pechukas, P.; McLafferty, F. J. J. Chem. Phys. 1973, 58, 1622.

(18) Amini, A. M.; Herman, M. F. J. Chem. Phys. 1993, 99, 5087.

(19) Baym, G.; Mermin, D. J. Math. Phys. 1961, 2, 232.

(20) Truhlar, D. G.; Garrett, B. C.; Klippenstein, S. J. J. Phys. Chem. 1996, $100,12771$.

(21) Simon, B. Functional Integration and Quantum Physics; Academic: London, 1979.

(22) Predescu, C.; Doll, J. D. J. Chem. Phys. 2002, 117, 7448. 